\title{
Guías para el manejo de la oxigenoterapia domiciliaria en pediatría. Parte 1: Generalidades, indicaciones y monitoreo
}

Guidelines for home oxygen therapy management. Part 1: Generality, indications and monitoring

Comité Nacional de Neumonología, $S A P^{*}$

http:/ /dx.doi.org/10.5546/aap.2013.448

\section{INTRODUCCIÓN}

El oxígeno $\left(\mathrm{O}_{2}\right)$ forma parte de la atmósfera natural en la que los seres humanos crecemos y nos desarrollamos.

La administración de $\mathrm{O}_{2}$ en los niños y adultos tiene un papel fundamental en el tratamiento de la hipoxemia y previene las complicaciones asociadas con la hipoxia tisular que se produce en la insuficiencia respiratoria crónica.

La hipoxemia se define como una tensión de $\mathrm{O}_{2}$ arterial $\left(\mathrm{PaO}_{2}\right)$ por debajo de la normal para la edad. Existe hipoxia cuando la tensión de $\mathrm{O}_{2}$ dentro de la mitocondria es inadecuada para mantener el metabolismo aeróbico. La hipoxemia medida a través de la $\mathrm{PaO}_{2}$ o la oximetría de pulso $\left(\mathrm{SpO}_{2}\right)$ constituye la principal indicación de oxigenoterapia, mientras que el principal objetivo de la oxigenoterapia es evitar o revertir la hipoxia tisular.

La administración de $\mathrm{O}_{2}$ suplementario continuo en pacientes adultos con enfermedad pulmonar obstructiva crónica (EPOC) ha demostrado ser eficaz no sólo porque mejora la calidad de vida, sino también porque aumenta la
Correspondencia: Dra. Verónica Giubergia: verogiubergia@gmail. com

Conflictos de intereses: Ninguno que declarar.

Recibido: 29-5-2013 Aceptado: $30-7-2013$
Secretaria del Comité: Verónica Giubergia.

Coordinadores: Fernando Vila, Claudio Castaños.

Participaron en la discusión y redacción de esta guía los siguientes miembros del Comité de Neumonología: Giubergia Verónica; Vila Fernando; Aguerre Verónica; Pawluk Victor; Dalessandro Virginia; Pereyro Silvia; Nadeo Julio; Paba Patricia; Castaños Claudio; Luque Graciela; Moncada Karina; Bonina Ángel; Moro Leonor; Urrutigoiti Jorge; Inwentarz Sandra; DiTulio Nicolás; Álvarez Daniel; Szulman Gabriela; Magadan Corina; Cracovsky Gabriel; Bodas Pablo; Balinotti Juan; Giorgetti Mariano; Smith Silvina; Ginestet María Eugenia; Andreottola María Elena; Rentería Fernando; Abram Lina; Martinchuck Migliazza Gisela; Fraga Marcela; Benítez Araceli; Baratta Sandra; Melillo Karina; Lombardero Lorena; Robles Raúl; Massa Sabrina; Adot Fernando; Cappelino Marcela; Barria Sandra; Castineiras Ana; Fiamingo Alfio; Petti Daniela; Tucci Nadia; Reches Beatriz; Fleitas Hugo; Bisero Elsa; Dagnino Diego; Kruger Anahi; Finochiaro Juan; Olagaray Lucas; Borda Mauricio; Taire Damián; Cipriani Silvina; Bujedo Elizabeth; Moreno Laura; Lepera Cecilia; Parra Luis; Lubovich Silvina; Salim Maxi; Pisapia Néstor; Toloza Rodolfo; Meneghuzzi Alejandra; Gallardo Liliana; Razovich Laura; Bonifachich Elena; Diaz Nora; Pierini Judith; Solis Teresa. 
expectativa de vida a largo plazo (especialmente cuando el $\mathrm{O}_{2}$ se utiliza al menos 15 horas diarias).

Durante la década de 1970, Neff y Petty, relacionaron la disminución de la mortalidad de los pacientes con EPOC con el uso de $\mathrm{O}_{2}$ suplementario, la reducción de la masa eritrocitaria y la disminución de la presión de la arteria pulmonar. Posteriormente otros trabajos confirmaron estos hallazgos. ${ }^{1}$

La oxigenoterapia en lactantes y niños mayores ha cambiado en los últimos 25 años desde una terapia empírica limitada, hasta una variedad de técnicas para su uso con bases científicas bien establecidas. La cantidad de niños que reciben oxigenoterapia domiciliaria (OTD) ha aumentado en los últimos años.

La eficacia y la seguridad en la terapia con $\mathrm{O}_{2}$, tanto en forma aguda como crónica, han mejorado significativamente desde el momento en que el $\mathrm{O}_{2}$ como cualquier otro fármaco, se prescribe y administra a una dosis determinada con monitoreo de los efectos adversos y de la toxicidad.

En los últimos años, se han desarrollado técnicas de monitoreo sencillas y no invasivas, especialmente en los lactantes, con una disminución significativa de las complicaciones y la toxicidad asociadas al uso del $\mathrm{O}_{2}$.

\section{DEFINICIÓN}

El término OTD, en el contexto en el que se utiliza en este consenso, se refiere a la utilización suplementaria de $\mathrm{O}_{2}$ fuera del hospital con la finalidad de mantener la $\mathrm{SpO}_{2}$ deseada en pacientes con hipoxemia crónica.

Los requerimientos pueden ser continuos (durante las 24 horas) o sólo intermitentes durante el sueño y la alimentación. ${ }^{2}$

\section{ALGUNAS CONSIDERACIONES}

\section{- Diferencias entre la práctica pediátrica y la} del adulto

- En general, las enfermedades pediátricas tienden a mejorar con el tiempo. En cambio, en los adultos, su condición es de progresivo deterioro. La excepción la constituyen los niños con fibrosis quística y enfermedades neuromusculares.

- En los niños es importante considerar el impacto de la hipoxemia en el crecimiento y el neurodesarrollo.

- El equipamiento es diferente. En los niños se requieren flujímetros milimetrados para asegurar flujos bajos.
- En cuanto al cuidado y la seguridad, requieren supervisión de un mayor de edad.

- Se debe asegurar su provisión cuando asisten al colegio.

- En pediatría, tanto la indicación como el monitoreo del $\mathrm{O}_{2}$ domiciliario se realizan con oxímetro de pulso y no con gases en sangre.

\section{- Valores normales de saturometría en} pediatría

- En los niños menores de 1 año (nacidos a término), la media basal oscila entre $95 \%$ y $98 \%$. Un $5 \%$ de estos niños presentan una $\mathrm{SpO}_{2}$ $<90 \%$ por un $4 \%$ del tiempo durante las 24 horas.

- A partir del año de vida, el percentil 50 de saturación es de $98 \%$ (el percentil 5 es entre $96 \%$ y $97 \%$ ).

- Entre los 5 y los 11 años, no más del 5\% del tiempo la saturación está debajo del $94 \%$ mientras están despiertos.

\section{- Consecuencias de una $\mathrm{SpO}_{2}$ baja crónica en} los niños

- Hipertensión pulmonar (HTP): niveles de $\mathrm{SpO}_{2}$ entre $88 \%$ y $90 \%$ pueden causar HPT, que se evita si la saturación se encuentra entre $94 \%$ y 95\%.

- Alteraciones en el neurodesarrollo: en diferentes revisiones sistemáticas, los niveles de $\mathrm{SpO}_{2}$ inferiores a $85 \%$ han demostrado efectos en la esfera cognitiva de los niños. Con niveles de $\mathrm{SpO}_{2}$ entre $85 \%$ y $90 \%$ estos efectos son menos claros y, por encima de $92 \%$, no se han detectado alteraciones. ${ }^{3,4}$

- Aumento del riesgo de apneas, muerte súbita y alteraciones de la calidad del sueño: se aprecia en los niños con enfermedad pulmonar crónica neonatal (EPCN) con $\mathrm{SpO}_{2}<90 \%$. Estos hallazgos no se observan con $\mathrm{SpO}_{2}>93 \%$.

- Crecimiento: en los niños con EPCN, una $\mathrm{SpO}_{2}$ $<92 \%$ puede asociarse con disminución del crecimiento.

\section{- Consecuencias del exceso de la terapia} con $\mathrm{O}_{2}$

Se ha observado que una elevada concentración de $\mathrm{O}_{2}$ a nivel arterial e intraalveolar puede ser tóxica en pacientes prematuros con DBP o sin ella. En el período neonatal, si la $\mathrm{SpO}_{2}$ es $>96 \%$ los niños pueden desarrollar con mayor frecuencia retinopatía del prematuro y se inhiben los mecanismos de cicatrización pulmonar. Los niveles de $\mathrm{SpO}_{2}$ óptimos sugeridos 
para este grupo etario deben encontrarse entre $93 \%$ y $95 \%$.

\section{OBJETIVO DE LA INDICACIÓN}

El objetivo de la indicación de OTD es corregir la hipoxemia. Hay muy poca evidencia de cuál es la $\mathrm{SpO}_{2}$ mínima recomendada. $\mathrm{La} \mathrm{SpO}_{2}$ normal es $\geq 96 \%$ y el $\mathrm{O}_{2}$ suplementario generalmente es recomendado en los pacientes que no mantienen saturaciones $\geq 93 \%$ Se recomienda certificar la $\mathrm{SpO}_{2}$ durante el ejercicio, la alimentación y el sueño.

\section{INDICACIONES DE OXIGENOTERAPIA DOMICILIARIA EN PEDIATRÍA}

La indicación de OTD en pediatría debe ser realizada por un pediatra neumólogo o, en su defecto, por pediatras o neonatólogos entrenados en la atención de pacientes con insuficiencia respiratoria crónica. Estos profesionales deberán estar a cargo del seguimiento y de evaluar el momento adecuado para la suspensión de la OTD.

La evaluación de la $\mathrm{SpO}_{2}$ debe realizarse con cuidado porque de ella depende la indicación de OTD. ${ }^{2}$

a. Enfermedad pulmonar crónica neonatal (la DBP es la más frecuente; otras menos frecuentes son: hipoplasia pulmonar, neumonía connatal, hernia diafragmática, aspiración de líquido meconial, etc.).

En este grupo de pacientes, la OTD debe indicarse para prevenir la HTP y mejorar el desarrollo neurológico, así como para disminuir el riesgo de muerte súbita, mejorar el crecimiento pulmonar y somático, reducir la posibilidad de infecciones hospitalarias y el impacto psicológico de la internación prolongada.

Los niveles deseados de $\mathrm{SpO}_{2}$ en este grupo se encuentran entre $93 \%$ y $95 \%$.

En los niños prematuros con DBP, el nivel de $\mathrm{SpO}_{2}$ indicado es de $93 \%$ a $95 \%$ y no debe superar en ningún caso este valor hasta completar la vascularización de la retina (44 semanas de edad posgestacional), dado que niveles mayores que estos están vinculados al desarrollo de la retinopatía del prematuro.

Estos niveles de $\mathrm{SpO}_{2}$ en los niños prematuros resultan de dos trabajos importantes: el BOOST y el STOP-ROP. El primero muestra que mantener una $\mathrm{SpO}_{2}$ entre $95 \%$ y $98 \%$ no tiene ventajas sobre niveles de $91 \%$ a $94 \%$ en función del crecimiento o el neurodesarrollo, mientras que el segundo muestra que $\mathrm{SpO}_{2}$ más elevadas producen mayor posibilidad de retinopatía del prematuro, sobre todo hasta las 44 semanas de edad posgestacional. ${ }^{4,5}$

b. Enfermedad intersticial. Debería considerarse la OTD en estos pacientes con el objetivo de mejorar la calidad de vida y la tolerancia a la actividad física, y prevenir la HTP.

En pediatría no hay estudios con niveles adecuados de evidencia en relación con la OTD en la neumonía intersticial crónica. En los adultos, un solo estudio no mostró beneficio de OTD en relación con la mortalidad después de tres años de tratamiento. ${ }^{7}$

Basado en la opinión de expertos, se recomienda ofrecer la OTD a estos pacientes.

c. Bronquiolitis obliterante (BO). $\mathrm{El}_{2}$ es una indicación en los niños con $\mathrm{BO}$ e hipoxemia. La mayoría de las BO son secundarias a infecciones respiratorias graves. Dado que no existe una terapéutica específica para esta enfermedad, muchos de estos niños requieren OTD prolongada.

En la experiencia del Hospital Garrahan, el $64 \%$ de los niños con BO posinfecciosa requirieron $\mathrm{O}_{2}$ luego de la infección, que pudo suspenderse en alrededor del 50\% de los pacientes que continuaban en seguimiento. ${ }^{8} \mathrm{Se}$ han publicado experiencias similares también en Chile. Algunos de estos niños pueden volver a requerir $\mathrm{O}_{2}$ con el desarrollo puberal.

d. Fibrosis quística (FQ). En los niños con FQ los niveles de hipoxemia tolerables son más bajos que en los que tienen las patologías antes mencionadas. Se indica OTD con $\mathrm{SpO}_{2} \leq 90 \%$ respirando aire ambiente. En estos pacientes, antes de la colocación de $\mathrm{O}_{2}$ domiciliario, deben monitorearse los niveles de $\mathrm{CO}_{2}$.

La hipoxemia en los niños con FQ es un signo de enfermedad pulmonar grave y antes de la indicación de OTD, se deben intensificar todos los tratamientos habituales, así como descartar otras complicaciones propias de la enfermedad.

La OTD debe indicarse sólo para mejorar la calidad de vida y no tempranamente con el objetivo de retrasar la progresión de la enfermedad. Extrapolando las experiencias de pacientes adultos con EPOC, se propuso que la OTD podría mejorar la supervivencia; sin embargo, esto no se pudo demostrar. ${ }^{2}$

En los niños con FQ, la OTD ha demostrado mejorar la concurrencia al colegio y la resistencia al ejercicio, por lo que puede 
mejorar la calidad de vida. También se la ha utilizado sola o en combinación con ventilación no invasiva (VNI) para el tratamiento de la hipoxemia nocturna. ${ }^{9}$

En los niños con FQ candidatos a un trasplante pulmonar, debe indicarse la OTD especialmente para evitar la HTP y sus consecuencias. Salvo estos pacientes, la OTD se indica en forma paliativa, ya que la HTP es muy poco frecuente.

e. Apnea obstructiva del sueño. Hasta que pueda resolverse quirúrgicamente en quienes es posible, la presión continua en la vía aérea (CPAP), u ocasionalmente la ventilación no invasiva (VNI), constituye la terapia de elección en la obstrucción de la vía aérea alta. Si esto no fuera factible, puede indicarse $\mathrm{O}_{2}$ domiciliario para mejorar la hipoxemia, siempre midiendo previamente los niveles de $\mathrm{CO}_{2}{ }^{10}$

f. Cardiopatías congénitas.

- Cianóticas: La OTD no debería usarse en los pacientes con cardiopatías congénitas cianóticas. La cianosis está producida por disminución del flujo sanguíneo pulmonar o por mezcla de la sangre pulmonar y la venosa, por lo cual el $\mathrm{O}_{2}$ tiene poco efecto en elevar la $\mathrm{SpO}_{2}$. En los casos de policitemia no está indicada la OTD, sin importar el nivel de la $\mathrm{SpO}_{2}{ }^{11}$

- Acianóticas: La OTD está indicada en presencia de una alteración ventilación/ perfusión por edema agudo de pulmón. Sin embargo, este es un cuadro que requiere hospitalización.

En los pacientes ya corregidos quirúrgicamente que cursan una infección respiratoria o luego de una intercurrencia aguda con insuficiencia respiratoria, debe evaluarse la indicación de OTD individualmente.

g. Hipertensión pulmonar (HTP). En la HTP idiopática primaria, el $\mathrm{O}_{2}$ suplementario está recomendado para la hipoxemia relacionada con el sueño por hipoventilación y en casos de emergencia (crisis de HTP). ${ }^{11}$

En los casos en los cuales la HTP es secundaria a una patología cardíaca relacionada con la inversión del flujo sanguíneo, el $\mathrm{O}_{2}$ suplementario no tiene ninguna indicación, ya que no corrige la hipoxemia.

h. Insuficiencia respiratoria secundaria a secuelas neurológicas. En los niños con secuelas neurológicas severas, la indicación de $\mathrm{O}_{2}$ debe evaluarse en forma individual.
En este grupo, deberían prevenirse las causas que llevaron a la insuficiencia respiratoria crónica y debería evaluarse si la corrección de la hipoxemia realmente mejora su calidad de vida y disminuye las intercurrencias respiratorias.

En estos pacientes, los niveles de $\mathrm{SpO}_{2}$ tolerados son menores que en otras patologías. Se debe indicar OTD en los pacientes con insuficiencia respiratoria crónica cuya $\mathrm{SpO}_{2}$ basal se encuentre en $88 \%$.

i. Enfermedades neuromusculares. Sólo un pequeño grupo de pacientes con enfermedades neuromusculares requieren OTD, y debe evaluarse en forma personalizada. En la mayoría de estos niños, la insuficiencia respiratoria se debe a una alteración de la bomba respiratoria y no está relacionada con el intercambio de gases a nivel pulmonar, por lo cual se benefician con la implementación de VNI.

j. Cuidados paliativos y final de la vida. No hay datos sobre el manejo de la disnea en el final de la vida en los niños. A pesar de ello, se usa el $\mathrm{O}_{2}$ en la etapa final de algunas enfermedades. En un estudio doble ciego realizado en adultos con cáncer en etapa terminal, la provisión de $\mathrm{O}_{2}$ por máscara mejoró la sensación subjetiva de disnea. ${ }^{12} \mathrm{Sin}$ embargo, un metanálisis reciente no mostró ningún beneficio. ${ }^{13}$

En estos niños probablemente sea más útil el uso adecuado de otras estrategias para disminuir la sensación de disnea, como el uso de opiáceos, en lugar de la OTD.

\section{TIEMPO DE UTILIZACIÓN}

Los beneficios de la OTD están directamente relacionados con el número de horas/día en que el paciente la utiliza. En general, la provisión de $\mathrm{O}_{2}$ se realiza durante las 24 horas (OTD continua), pero en los casos que corresponda se realizará sólo durante el sueño (OTD relacionada con el sueño) o durante la noche (OTD nocturna). ${ }^{2}$

En los pacientes que usan OTD continua, se debe estimular a que el paciente reciba $\mathrm{O}_{2}$ la mayor cantidad de tiempo posible; si bien lo óptimo son 24 horas, debe recibir al menos 15 horas por día, incluidas las horas del sueño y de la alimentación. ${ }^{14}$

\section{MONITOREO}

La determinación en una muestra de sangre arterial del $\mathrm{pH}, \mathrm{PaO}_{2}, \mathrm{PaCO}_{2}$ y $\mathrm{SpO}_{2}$ y su 
evaluación a través de un analizador de gases en sangre es el método de elección para determinar los niveles de $\mathrm{O}_{2}$ sanguíneo y el más adecuado en los niños aguda y críticamente enfermos. Las muestras pueden obtenerse a través de la punción arterial directa o por catéteres colocados por vía percutánea.

Las muestras de sangre capilar arterializada obtenidas en los lactantes (por calentamiento y punción del área) tienen regular correlación con las muestras arteriales en relación con el $\mathrm{pH}$ y la $\mathrm{PaCO}_{2}$; tampoco son adecuadas para evaluar la oxigenación.

Esta evaluación es fundamental para caracterizar la insuficiencia respiratoria del paciente, evaluando los niveles de $\mathrm{PCO}_{2}$ que este maneja. Se debe realizar al indicar la OTD en el centro de mayor complejidad. No está indicada para controlar la evolución del paciente en el domicilio.

En los pacientes con OTD prolongada y enfermedad grave, se recomienda obtener una muestra de gases en sangre una vez por año.

\section{Sistemas de evaluación de $\mathrm{SpO}_{2}$}

Existen dos sistemas no invasivos para evaluar la oxigenación arterial que aportan información continua sin la necesidad de obtener muestras de sangre. Son muy confiables en los pacientes que tienen una función cardiovascular normal.

a. Monitor transcutáneo de $\mathrm{O}_{2}$ (actualmente en desuso).

b. Oximetro de pulso: es la forma más simple y segura de monitorear la oxigenación del paciente en pediatría. Puede usarse a toda edad y no requiere calibración. Es el método de elección para la indicación y el monitoreo de la OTD. ${ }^{15}$ Existen distintos tipos de oxímetros. Algunos tienen rangos diferentes de captación, por lo cual pueden subestimar o sobreestimar las lecturas de $\mathrm{SpO}_{2}$.

\section{Características del sistema}

- Técnicamente fácil de usar e incruento.

- Fundamento técnico: el dispositivo emite luz con dos longitudes de onda. La mayor parte de la luz es absorbida por el tejido conjuntivo, la piel, el hueso y la sangre venosa en una cantidad constante, produciéndose un pequeño incremento de esta absorción en la sangre arterial con cada latido, lo que significa que es necesaria la presencia de pulso arterial para que el aparato reconozca alguna señal. La transmisión de la luz a través de la hemoglobina es directamente proporcional a la $\mathrm{SpO}_{2}$.

- Detecta la hipoxemia antes de que se aprecie cianosis, ya que esta suele observarse cuando la $\mathrm{PaO}_{2}$ cae por debajo de $60 \mathrm{~mm} \mathrm{Hg}$, lo que corresponde a una $\mathrm{SpO}_{2}$ aproximada de $90 \%$.

- Se debe seleccionar un sensor adecuado al tamaño del niño y colocar en una zona limpia con buena irrigación y temperatura. Los sitios adecuados para la colocación del sensor son los pulgares de las manos o los pies, y los lóbulos de las orejas.

- La forma correcta es colocar el sensor que corresponda de acuerdo con la edad del paciente y esperar el registro de la $\mathrm{SpO}_{2}$ hasta que no fluctúen los valores, corroborando con la auscultación la frecuencia cardíaca (FC). La FC se debe correlacionar con la detectada por el oxímetro y registrar este valor junto con la FC, la temperatura y el sitio donde se colocó el sensor.

- Es necesario obtener una onda de pulso de morfología normal y sin alteraciones de la frecuencia cardíaca.

- No requiere calibración frecuente.

- Se deben colocar los sensores adecuados de acuerdo con la edad del paciente.

\section{Limitaciones}

- La señal se pierde cuando existe hipoperfusión.

- Puede haber interferencia con otros equipos.

- No diferencia entre oxihemoglobina, carboxihemoglobina y metahemoglobina, y puede haber errores cuando la hemoglobina presenta alguna de estas formas.

- No detecta cambios de $\mathrm{SpO}_{2}$ hasta que la $\mathrm{PaO}_{2}$ cae entre 70 y $80 \mathrm{~mm} \mathrm{Hg}$.

- No reemplaza los gases arteriales $\left(\mathrm{pH}, \mathrm{PaO}_{2^{\prime}}\right.$ $\mathrm{PCO}_{2}$ y $\mathrm{SpO}_{2}$ ).

- No evalúa el proceso de ventilación del paciente, ya que no mide la $\mathrm{PCO}_{2}$.

- Se debe evitar tener las uñas pintadas, ya que los esmaltes contienen acrílico, que altera la lectura.

- También se deben evaluar las fallas derivadas de la postura inadecuada del sensor y de la exposición a la luz ambiente (lámparas, luz fluorescente, fibras ópticas), y evitar los movimientos del paciente, ya que todas estas causas alteran los resultados.

\section{Correlación entre la $\mathrm{SpO}_{2}$ y la $\mathrm{PaO}_{2}$}

La correlación entre la saturación de $\mathrm{O}_{2}$ y la $\mathrm{PaO}_{2}$ está determinada por la curva de disociación de la oxihemoglobina (Figura 1). 
Una manera simple de valorar la relación entre la $\mathrm{PaO}_{2}$ y la $\mathrm{SaO}_{2}$ en forma aproximada es la siguiente:

\begin{tabular}{cc}
$\mathrm{SaO}_{2}$ & $\mathrm{PaO}_{2}$ \\
\hline $70 \%$ & 40 \\
$80 \%$ & 50 \\
$90 \%$ & 60 \\
\hline
\end{tabular}

En los pacientes que se reciben OTD, se evalúa la $\mathrm{SpO}_{2}$ a través del oxímetro de pulso. El control inicial se realiza con el mismo aporte de $\mathrm{O}_{2}$ suplementario administrado a través de la cánula nasal con el que el niño se maneja en su casa.

En los niños estables clínicamente, que requieren flujos cada vez menores para mantener una $\mathrm{SpO}_{2} \geq 93 \%$, en quienes se decidió evaluar la posibilidad de retirar la administración de $\mathrm{O}_{2}$, este debe suspenderse por 20 minutos y realizar un nuevo control. Se registran ambos valores y también los eventos ocurridos en el transcurso del control: llanto, sueño, alimentación o inquietud, frecuencia cardíaca y respiratoria.

En los pacientes con enfermedad grave que requieren flujos altos para mantener una $\mathrm{SpO}_{2}$ $\geq 93 \%$, sólo se realiza el control de esta cuando están recibiendo $\mathrm{O}_{2}$. La suspensión del $\mathrm{O}_{2}$ en estos niños puede provocar episodios de hipoxemia grave.

Cuando se encuentran en plan de suspensión de la OTD, se debe registrar el tiempo en horas del retiro del $\mathrm{O}_{2}$ y la $\mathrm{SpO}_{2}$. Si esta fuera $\leq 92 \%$, se debe administrar nuevamente, por cánula nasal comenzando con $0,5 \mathrm{~L} / \mathrm{min}$ y aumentando progresivamente hasta lograr una $\mathrm{SpO}_{2} \geq 93 \%$.

No hay ninguna evidencia que indique que la permanencia del saturómetro en el domicilio del paciente sea beneficiosa o perjudicial, y debe evaluarse en cada caso. ${ }^{2}$

\section{BIBLIOGRAFÍA}

1. Neff TA, Petty TL. Long-term continuous oxygen therapy in chronic airway obstrucción: mortality in relationship to cor pulmonale, hypoxia and hypercapnia. Ann Intern Med 1970;72:621-6.

2. Balfour-Lynn IM, Field DJ, Gringras P, Hicks B, Jardine E, et al. on behalf of the Paediatric Section of the Home Oxygen Guideline Development Group of the BTSStandards of Care Committee.BTS guidelines for home oxygen in children. Thorax 2009;64 (Suppl II):1-26.

3. Urschitz NS, Wolff J, Sokolik C, Eggebrecht E, et al. Nocturnal arterial oxygen saturation and academic perfomance in a comunity sample of children. Pediatrics 2005;115:e204-9.

4. Askie LM, Henderson-Smart DJ, Irwig L, Simpson JM. Oxygen saturation targets and outcomes in extremely preterm infants. N Eng J Med 2003;349:959-67.

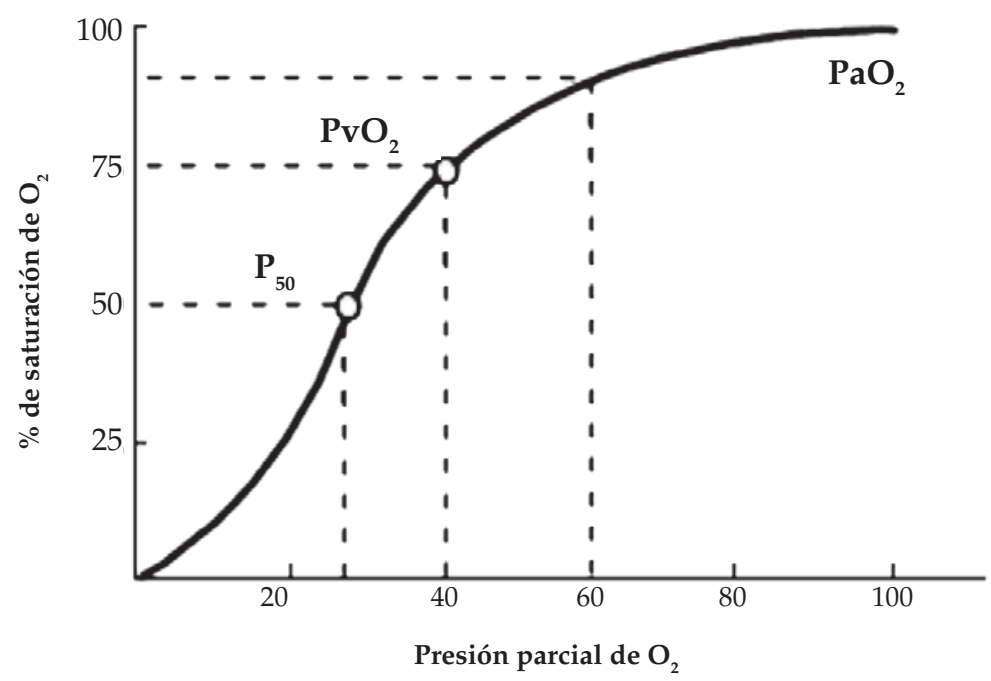

La curva de disociación de la hemoglobina es plana a niveles altos de $\mathrm{PaO}_{2}$ y la $\mathrm{SaO}_{2}$ no disminuye significativamente hasta que la $\mathrm{PaO}_{2}$ es de 75-78 mmHg. 
5. The STOP-ROP Multicenter Study Group. Supplemental Therapeutic Oxygen for Prethreshold Retinopathy Of Prematurity (STOP-ROP), a randomized controlled Trial. I: primary outcomes. Pediatrics 2000;105:295-310.

6. Weinberger B, Laskin DL, Heck DE, Laskin JD. Oxygen toxicity in premature infants. Toxicol Appl Pharmacol 2002;181:60-7.

7. Crockett A, Cranston J, Antic N. Domiciliary oxygen for interstitial lung disease. Cochrane Database Syst Rev 2001;3:CD002883.

8. Murtagh P, Giubergia V, Viale D, Bauer G, Pena HG. Lower respiratory infections by adenovirus in children. Clinical features and risk factors for bronchiolitis obliterans and mortality. Pediatr Pulmonol 2009;44(5):450-6.

9. Zinman R, Corey M, Coates AL, Canny GJ, et al. Nocturnal home oxygen in the treatment of hypoxemic cystic fibrosis patients. J Pediatr 1989;114:368-77.

10. Aljadeff G, Gozal D, Bailey-Wahl SL, Burrel B. Effects of overnight supplemental oxygen in obstructive sleep apnea in children. Am J Respir Crit Care Med 1996;153:51-5.

11. Widlitz A, Barst RJ. Pulmonary arterial hypertension in children. Eur Respir J 2003;21:155-76.
12. Bruera E, de Stoutz N, Velasco-Leiva A, Schoeller T, et al. Effects of oxygen on dyspnoea in hypoxaemic terminalcancer patients. Lancet 1993;342:13-4.

13. Uronis HE, Currow DC, McCrory DC, Samsa GP, et al. Oxygen for relief of dyspnoea in mildly-or non-hypoxaemic patients with cancer: a systematic review and meta-analysis. Br J Cancer 2008;98:294-9.

14. MacLean JE, Fitzgerald DA. A rational approach to home oxygen use in infants and children. Paediatr Resp Rev 2006;7:215-22.

15. Balfour-Lynn IM, Primhak RA, Shaw BNJ. Home oxygen for children: who, how and when? Thorax 2005;60:76-81.

16. ANMAT. (Consulta 10 de Abril de 2013). Disponible en http//: www.anmat.gov.ar.

17. Thoracic Society Standards of Care Committee Managing passengers with respiratory disease planning air travel: British Thoracic Society recommendations. Thorax 2011;66:130.

18. AguerreV.Viajeen avión, riesgos y previsiones a considerar en los niños. Arch Argent Pediatr 2012;110(1):66-9. 